\title{
Ethique et formation médicale Révision des concepts
}

Guy LLORCA*

M essages Clés • L'éthique médicale doit être enseignée • L esintentions pédagogi ques sont centrées sur l'amélioration de la qualité des pratiques et de la relation de confiance. - Cet enseignement doit être adapté aux différentes cibles représentées par les médecins en formation initiale, les médecins en formation continue, les formateurs en éthique médicale et les chercheurs en éthique médicale. • En formation initiale, l'objectif principal et d'apprendre le raisonnement éthique • En formation continue, l'analyse de cas réels est certainement primordiale • La formation des formateurs doit apporter les connaissances fondamentales en éthique et en animation de groupe. $\bullet \mathrm{L}$ a formation des chercheurs doit comporter l'approche des méthodes qual itatives - L'éthique de l'ensei gnement repose sur trois valeurs fondamentales : la liberté, la justiceet la responsabilité. • L'éhique del'enseignement aboutit à un contrat entre formateurs, formés et institution. - Son respect est d'uneévaluation délicate et doit privilégier les démarches formatives fondées sur uneacceptation générale et libre desacteurs.

Mots clés Éthique ; Bioéthique ; Éthique médicale ; Éthique Clinique ; Comité d'éthique ; Enseignement M édical ; Formation Initiale; Formation Continue; M odèles; M éthodes; Techniques; Évaluation.

Key messages • M edical thics must beteached. • Teaching design iscentred on improvement of medical practice and confidence relationship. - M edical ethi cs teaching must be adjusted to different targets: undergraduates, postgraduates, teachers and researchers. - For undergraduates, the main aim is to learn éthical reasoning. - For postgraduates, solving real thical problem is certainly the leading purpose - Teachers must be competent in fondamental ethics and group management. • Reaserchersmust learn quali tatives methods. - Ethics of medical education, is based on three principal values: liberty, justice and responsability. - Ethics of medical education leads to a three part-contract established between teachers, sudents and institution. - The evaluation of application of thiscontract must be necessarly founded in a free and consent acceptation of all the actors

Key Words Ethics; Bioethics; M edical Ethics; Clinical Ethics; Ethics Committee; M edical Education; Undergraduate; Postgraduate; M odels; M ethods; Techcnics, Evaluation. Pédagogie M édicale 2002; 3 : 169-179

\section{Introduction}

La seconde moitié du $x x^{e}$ siècle a été marquée par de profonds bouleversementsqui n'ont pasmanquédetoucher la médecine. Bouleversements techno-scientifiques, en premier lieu, qui sont à l'origine d'une médecine délibérément efficace et d'une approche scientifique de la décision médicale, soutenues par la démarche fondée sur des preuves contenue dans le concept d'evidence-based medicine ${ }^{1,2}$. Bouleversements socioculturels et idéologiques, en second lieu, à l'origine de nouvelles représentations de la vie et de la mort, de la santéet de la maladie, du soin et des traitements préventifs, curatifs ou palliatifs...

Alors que la médecine avait centré ses valeurs sur la religion et la loi, ces bouleversements sont à l'origine d'un immense phénomène social et intellectuel qui se concré tise avec la nai ssance du terme bioéthique crééen 1971 par I'oncologiste Van Rensselaer Potter en publiant son livre intitulé « Bieothics Bridge to the Future » et celui d'éthique clinique, créé en 1976 par Joseph Fletcher 3.4 . L'éthique médicale, qui se construit à partir dela philosophie morale et dela déontologiedela profession, tentederépondreaux

\footnotetext{
* Comité d'Ehique du CHU de Lyon, France.

Correspondance : Pr. G Llorca, Laboratoire de Thérapeutique, 8, avenue Rockefeller 69373 Lyon cedex 08 France Téléphone : (33) 478777063 - Télécopie : (33) 478014934

Mailto:guy.llorca@chu-lyon.fr
} 


\section{Références}

nouvelles questions et préoccupations. En effet, en même tempsquel'espérance de vieprogresse, la notion de qualité de vie reliée à la santé se développe et apparaît comme un objectif médical majeur. Ces bouleversements sont à l'origine de tensions relationnelles au sein de sociétés devenues multiculturelles qui mettent en cause l'asymétrie des pouvoirs traditionnels.

Les années 60 sont marquées par le rôle important de théologiens et de philosophes qui sont sollicités par la prolifération des biotechnologies, les abus de la recherche et l'apparition d'une nouvelle conscience des patients, de leurs droits et des responsabilités. Un fort mouvement est initié, dans les années 70, en Amérique du N ord avec, en particulier aux Etats- $U$ nis, la création du K ennedy Institute of Ethics à I'université G eorgetown de Washington en 1971, la National Commission on the protection of $\mathrm{H}$ uman Subjects of Biomedical and Behavioral Research en 1974, la President's Commission for Study of Ethical Problemsin M edicineand Biomedical and Behavioral Research en 1979. D es Comités d'éthique et des consultations d'éthique sont mis en place et des médecins et philosophes créent un certain nombre de centres (dont le $\mathrm{H}$ astings $\mathrm{C}$ enter à $\mathrm{N}$ ew York, le Kennedy Institute à W ashington, le $\mathrm{M}$ ac Lean Center à Chicago, I'Institute for the M edical H umanities à G alveston... ). Ces centres sattaquent au problème de l'enseignement, des livres et revues spécialisés sont édités, des sociétés d'éthique sont créées... Ce mouvement s'étend rapidement au C anada et à l'Europe, puis à l'Asie et l'Afrique, essentiellement sous la forme deC omités d'éthiqueet dela création denombreux diplômes à forte consonance philosophique le plus souvent. D e ce mouvement international et des courants qu'il génère naît une mosaïque de situations, en même tempsque la notion de pluridisciplinarité devient évidente et enrichit la pensée médicale.

C'est dans ce climat de profonde mutation de la communication humaine, derévision des concepts clés dont celui de la santé et de l'utilité collective, d'évolution des croyances et del'habitat que la médecine doit progresser, si elle veut demeurer crédible. Sa finalité même doit être redéfinie pour ne pas dévier vers une obligation de résultat que la médiatisation des progrès et des échecs médicaux incite à introduire. Cette finalité est confrontée, non plus àla seule souffrance humaine, mais aux nouvelles maitrises psychiques et génétiques et à leurs détournements possibles. Elleest mal menée par les impératifs soit naturaliste, soit technicien qui s'affrontent et tentent de répondre à la question : « tout ce qui est techniquement possible est-il éthique? ». L'évolution des dernières années tend à remplacer le concept de santé par celui, plus raisonnable, de « potentiel-santé » et cherche à répondre aux positions extrêmes, technophobes et technophiles, par une démarche «prudentialiste ", c'est-à-dire encadrée de critères fixant les limites collectivement les plus acceptables. D ans la formation médicale, l'éthique apparaît maintenant comme un atout majeur et incontournable $e^{5,67.7}$.

Ce travail est consacré à deux aspects fondamentaux de l'éthiqueen formation médicale. Tout d'abord celui deson enseignement, en proposant un système modulaire susceptible de couvrir les divers besoins en la matière. Ensuite, celui de l'éthique de l'enseignement, qui est centrée sur des valeurs qui méritent d'être rappelées et soulèvent la question de leur mise en pratique.

\section{L'enseignement de l'éthique}

Ce premier chapitre tente de proposer un ensemble cohé rent d'enseignement de l'éthique médicale aux médecins, non pas pour privilégier ce corps professionnel, mais parce qu'il est le premier concerné, et parce qu'à partir de cette réflexion il est relativement ai sé de transposer ces propositions en les adaptant aux besoins et nécessités des autres professions de santé. Cette proposition, établie à partir des nombreuses expériences internationales, cherche à répondre aux difficultés qui ont été mises à jour par ces tentatives ${ }^{6.15}$. Ainsi, cet enseignement doit pouvoir correspondre aux attentes, tant en matière de formation initiale que continue.

\section{D éfinitions et domaines del'éthique médicale}

L'éthique médicale, composante forte de l'éthique, ne la résume pas. Elle emprunte ses bases à ce qu'il est convenu d'appeler l'éthique fondamentale ${ }^{16,17}$ et son approche pratique différencie le domaine clinique ${ }^{18}$ de celui de la recherche biomédicale et des techniques ${ }^{19}$. Si I'on se rapporteà la définition qu'en donne Reich ${ }^{20}$, la bioéthiqueest «... l'étude systématique de la dimension morale (incluant la vision morale, les décisions, la conduite et les politiques) des sciences de la vie et de la santé, utilisant les variétés de méthodeséthiques dans une approche multidi sciplinaire.. ». En ce qui concerne strictement la formation médicale, l'éthique médicale et singulièrement l'éthique clinique, doit être centrée, à notre point de vue sur le jugement de valeur de la décision médicale pratique ${ }^{7,21}$. 
O bligatoirement pluridisciplinaire, elle n'appartient pas strictement aux médecins mais les concerne en premier lieu. Ainsi recentrée, l'éthique médicale peut, plus facilement, pénétrer la formation de tout médecin par le biais de son domaine clinique et peut concerner, au titre des techniques et de la recherche, ceux qui souhaitent approfondir leur formation ou devenir, eux-mêmes, formateurs ou chercheurs en éthique médicale.

\section{Intentions pédagogiques}

Pour demeurer dans le cadre d'une finalité du soin centrée sur la qualité du conseil et de l'aide prodigués par le soignant ${ }^{12,13,22}$, l'intention pédagogique générale est représentée par l'aide apportée au médecin pour toujours privilé gier le meilleur (ou le moins mauvais) chemin pour répondre à la demande des personnes qui le sollicitent. II sagit alors, d'améliorer la qualité de la pratique médicale, en optimisant les propositions d'action, donc de décision, et en favorisant la compétence des soignants et la sécurité des soignés. Cetteintention de qual ité décisionnelles'associe à une intention de renforcement de la relation de confiance, par l'apprentissagedu respect detous les acteurs impliqués dansla décision médicale et celui des conditions d'une véritable communication.

\section{L'enseignement de base en formation initiale: le minimum indispensable}

\section{La cible pédagogique}

Elle est représentée par les étudiants en phase prédoctorale ou formation initiale. Elle ne doit pas faire perdre de vue que la cible véritable est représentée par le patient potentiel, et doit mettre en œuvre les méthodes les plus pertinentes pour assurer la cascade de redistribution des connaissances du formateur à l'étudiant, puis de l'étudiant devenu praticien au patient ${ }^{23,24}$.

\section{Les objectifs généraux}

Les objectifs généraux de formation, pour répondre aux intentions pédagogiques déclarées, doivent s'articuler autour de notions concrètes d'éthique clinique qui repré sentent les aptitudes de base que l'on souhaite voir acquérir et pouvoir évaluer à partir d'une modification comportementale induite observable. Six objectifs principaux nous semblent indispensables à ce stade de formation initiale:
1) savoir éval uer le degré de vulnérabilité d'un patient ;

2) savoir explorer les dimensions décisionnelles;

3) savoir identifier un conflit de valeur ;

4) savoir informer correctement un patient et son entourage;

5) savoir créer les conditions d'un vrai dialogue ;

6) savoir proposer une solution acceptable;

C es objectifs relèvent de compétences complexes et variées. Ils ne se situent pas tous au même niveau de connaissances et relèvent de savoirs multiples qui peuvent être sollicités à des moments différents du cursus médical.

\section{Les contenus}

Les contenus concernent les compétences entenduesau sens des savoirs, qu'ils soient factuels, conceptuels, procéduraux ou décisionnel $s^{25}$. Ces contenus sont obligatoirement pluridisciplinaires et ne sauraient être exhaustifs. Ils doivent être centrés sur la finalité de l'action médicale et doivent repré senter ce que tout médecin devrait connaître pour agir avec compétence. Ils ne doivent pas correspondre à des listes de réponses toutes prêtes à appliquer ou à une vérité révélée, mais aux instruments susceptibles d'aider à trouver la meilleure solution face aux difficultés rencontrées ${ }^{26-28}$. En fait, il apparait à l'analyse que, pour atteindre les objectifs généraux définis au paragraphe précédent, certains savoirs représentent des préalables, alors que d'autres apparaissent plus spécifiques de l'enseignement de l'éthique:

- les savoirs préalables : il s'agit, tout d'abord, des savoirs médicaux cliniques représentés par les connaissances sémiologiques, nosologiques, pronostiques et thérapeutiques sans lesquelles aucune décision médicale ne saurait être engagée II sagit, ensuite, des savoirs juridiques, psychologiques cognitifs, sociologiqueset économiquesqui seront habituellement sollicités par la réflexion éthiquedans son cadre décisionne habitue.

- Les savoirs spécifiques concernent les éléments indispensables à l'application des savoirs non spécifiques aux trois acteurs impliqués dans la décision médical eque sont le soignant, le soigné et la collectivité. C es savoirs spécifiques recouvrent trois domaines majeurs:

1) le domaine dela philosophiemoraleappliquéàl'éthique médicale (connaissances des règles, recommandations et principes de base qui doivent guider l'action et sont susceptibles d'entrer en conflit ${ }^{19}$ ) ;

2) le domaine de la communication éthique (particulière ment les critères de qualité de l'information honnête et les règles élémentaires du vrai dialogu $e^{29,30}$ ), 


\section{Références}

3) le domaine du raisonnement éthique (utilisant les textes de référence ${ }^{31,32}$ et les méthodes de résolution de conflit en la matière, avec les différents courants et modèles modernes recouvrant les principales sensibilités ${ }^{33-36}$ ).

Bien entendu, d'autres contenus peuvent compléter cet inventaire de base, notamment en matière d'épistémologie, d'anthropologie, d'ethnologie, de théologie, d'histoire, de linguistique, de sciences politiques etc. Ces compléments ne sont exigibles, dans la formation pratique d'un médecin, que sils doivent êtres souvent sollicités du fait de conditions particulières d'exercice et peuvent repré senter al ors des modules complémentaires ${ }^{37} \ldots$

Une liste de thèmes exemplaires, sources de difficultés et sujets d'études potentielles, peut être élaborée à partir des nombreuses expériences rapportées ${ }^{13,38-46}$. Ces sujets peuvent êtreabordés à partir du point de vue de chaqueacteur décisionnel ou, plus schématiquement, classés à partir de leurs sources, représentées par les personnes, les normes et la décision (cf. Tableau I).

\section{Les méthodes}

Les méhodes utiles à l'enseignement de l'éthique ne peuvent être que multiples, dépendant du niveau des stagiaires, des contenus sollicités et des objectifs poursuivis. D eux types de méthodes semblent cependant indispensables à utiliser selon que l'on se situe dans un contenu théorique discursif ou pratique procédural ${ }^{12,46,47}$.

- Les contenus théoriques peuvent être enseignés par des méthodes discursives traditionnelle ${ }^{11,15,24,48-50}$, ou bien laissés àl'initiative des stagiaires, ai dés de documents adéquats ou orientés vers des structures d'enseignement extra médicales dédiées à ces contenus, lorsqu'elles existent ${ }^{7,37}$. Une bibliographie de base doit être fournie aux étudiants pour leur éviter d'errer dans l'immense littérature que l'éthique médical ea suscitée. D es ouvrages d'une grande utilité existent maintenant, tant en langue anglaise ${ }^{51-54}$ qu'en langue françai se ${ }^{17,55,56}, 18$ publications périodiques dédiées ont été recensées par $\mathrm{H}_{\text {ottois }}{ }^{3}$ et les bases de données spécifiques informatisées peuvent représenter un outil complémentaire précieux ${ }^{57,58}$.

- Les contenus pratiques méritent de faire appel à des méthodes plus actives et susceptibles de mettre les stagiaires en situation 14,28,44,59-62. Trois types de méthodes peuvent répondre à ces besoins: 1- la simulation en ateliers d'apprentissage au raisonnent éthique (ARE), calqués sur les ateliers d'ARC ${ }^{63}$, privilégiela démarche de résolution de problème à partir del'analyse de cas, de préférence réels et ayant trouvé une solution consensuelle ; 2 - cette analyse peut aussi sintégrer dans le cadre de structures existantes dédiées, comme, par exemple, les Comités d'éthique locaux ${ }^{7}$; 3- elle doit, enfin, faire appel à un entraînement continu à l'occasion des stages cliniques hospitaliers ou au cabinet du praticien, en utilisant alors toutes les ressources du compagnonnage, de la réflexion de groupe et des techniques informatiques ${ }^{64,65}$ et vide $0^{66}$.

Il est évident que le choix de ces méthodes doit être guidé par les ressources locales et le niveau des stagiaires. Le volume horaire d'une telle formation de base est également fonction desconditions et possibilités locales, maisil semble qu'un minimum de 20 à 30 heures soit nécessaire pour présenter l'essentiel de la partie théorique et consacrer suffisamment de temps au travail interactif en groupes restreints. Le meilleur moment de cet enseignement n'est pas définitivement établi. Si la partie théorique peut être envisagéetrèstôt, l'entrainement au choix décisionnel doit certainement être plus tardif, faisant intervenir de nombreuses connaissances non spécifiques préelables.

\section{L'évaluation}

L'évaluation d'un tel enseignement est complexe car elle concerne des modifications comportementales et peut sintéresser aussi bien à l'approche décisionnelle médicale qu'à ses conséquences, et ce, dans un but de certification ou formatif $8,13,28,42,67,68,69,70$.

- D ans un but de certification, le plus délicat en la matière est de définir les normes de référence qui ne doivent pas représenter la vérité révélée obligatoire. Un consensus local peut être réalisé sur les contenus théoriques qui sont, alors, facilement explorés par des méthodes interrogatives classiques, rédactionnelles ou en réponses multiples automati sées. C ette évaluation peut valablement rendre compte des savoirs factuels, conceptuels, voire procéduraux, lorsqu'ils existent, mais se prête mal aux savoirs décisionnels. Les savoirs décisionnels ne semblent pouvoir être valablement explorés que par le biais d'approches critériées à partir des objectifs généraux décrits au paragraphe 0 bjectifs généraux, et, par exemple, par l'élaboration de cas éthiques simulés (CES) ou d'examens cliniques objectifs structurés $(E C O S)^{71,72}$. Les échelles de type Likert et l'approche de concordance de script peut valablement se substituer, ici, à la notation traditionnelle pour rendre mieux compte de l'élaboration de consensu ${ }^{66,73}$.

- D ans un but formatif, l'évaluation mérite d'être centrée sur les conséquences des comportements et peut utiliser 


\section{Tableau I : Liște indicative des thématiques sollicitées en Éthique Médicale et Clinique}

\begin{tabular}{|c|c|c|}
\hline Personnes et acteurs & Normes et règles & D écision et action \\
\hline Conflit d'intérêt & Autonomie (principe) & Abstention thérapeutique \\
\hline Comportement & Bénéfice individuel & Abus d'autorité, de pouvoir \\
\hline Consentement & Bienfaisance (principe) & Acharnement thérapeutique \\
\hline Contrôle & D élibération, Dialogue & Aléa thérapeutique \\
\hline Dignitéhumaine & D éontologie & Allocation des ressources \\
\hline D irectives anticipées & D ouble effet & Annonce d'une mauvaise nouvelle \\
\hline D ALE (D isability adjusted life & D ouble insu & Avortement \\
\hline expectancy) & Droit d'information & Clonage \\
\hline Empreinte génétique & D roit de ne pas savoir & Contraception \\
\hline Fin de vie & D roits del'homme, des enfants & Contrainte \\
\hline Incapable, Incompétent & D roits des malades & D opage \\
\hline Influence (detiers) & Équité & Erreur médicale \\
\hline Intégrité et sévices & Immortalité & Eugénisme \\
\hline M aladie orpheline & Impératifs & Euthanasie et suicide assisté \\
\hline M ort (critères, représentation) & Information & Expérimentation humaine \\
\hline Parentés nouvelles & Informatique médicale & Faute médicale \\
\hline Personne(définition, croyances) & Justice (principe) & Gestation pour autrui \\
\hline Personnalité & M aximes & Greffes d'organe, tissus, cellules \\
\hline Protection (vie privée, personne.) & M éthodologie bioéthique & Grossesse post-ménopausale \\
\hline Relation soignant-soigné & Non-malfai sance (principe) & IVG (interruption volontaire de grossesse) \\
\hline Répondant éthique & Paternalisme & M aternité de substitution \\
\hline QALY & Perte de chance & M édecine de convenance, désir \\
\hline Refus de soin, de traitement & Précaution, prudence (principe) & M édicament orphelin \\
\hline Respect del'autre & Principisme, principlisme & Transplantations \\
\hline Stade terminal & Q ualité de vie (critères) & PM A (procréation médicalement assistée) \\
\hline Suicide & Réciprocité (principe) & Prévention \\
\hline Testament de vie & Responsabilité (principe) & Risque (évaluation, inutile) \\
\hline Tiers compétent & Sacralité de la vie (principe) & Soins curatifs, palliatifs, prolongés \\
\hline Toxicomanie & Santé (définition, droit) & Sport et médecine \\
\hline Vérité(au malade) & Secret médical, confidentialité & Stérilisation \\
\hline Vie & Textes (nationaux, internationaux) & Tests (biologiques, génétiques) \\
\hline Vulnérabilité & Vertus, Valeurs & Traitement inutile, futile \\
\hline
\end{tabular}




\section{Références}

l'analyse de satisfaction des patients et la comparaison casuistique analogique (comparaison avec les modalités comportemental es qui avaient été retenues comme satisfaisantes de manière consensuelle pour un cas similaire). $D$ ans ce domaine, I'essentiel nous semble devoir se situer dans I'aptitude du stagiai re à documenter rationnellement son attitude, en démontrant qu'elle a toute probabilité d'aboutir à un accord général librement consenti51. D e manière plus pratique, l'évaluation de l'enseignement devrait porter sur des enquêtes de satisfaction des patients, sur des sondages d'opinion concernant l'évolution de l'image du corps médical, ou sur les résultats objectifs qui peuvent être mesurés à partir de l'évolution des taux de changement de comportements observables et à l'occasion d'audit de pratique.

\section{L'enseignement complémentaire et la formation continue}

L'enseignement continu post-doctoral est actuellement complexe du fait de la diversité des enseignements de base, lorsqu'ils existent, des intentions pédagogiques et des cibles viséés, $83,70,74,75,76$ :

\section{En formation médicale continue}

D ans ce cadre, il s'agit d'accroître les compétences et performances de professionnels dotés d'une expérience. Si l'enseignement de base a été réalisé dans le cursus des études médicales, la formation continue en éthique clinique ne se conçoit bien qu'en ateliers ou dans des structures d'échange entre professionnels avec, si besoin, participation d'experts des autres domaines concernés. Si l'enseignement de base n'a pas été dispensé au préalable, la formation continue doit opérer un rattrapage de manière à combler ce manque, en s'appuyant sur les objectifs déterminés au paragraphe précédent. Les contenus peuvent soit répondre à des situations réellement rencontrées par les participants, soit être élaborés à partir des thématiques spécifiques (cf. Tableau I) qui servent alors de base à une confrontation des expériences. Là aussi, I'approche de concordance de script ${ }^{73}$ peut repré senter non seulement une méthode d'évaluation mais aussi un moyen de parvenir à un accord général. L'évaluation de telles formations est, bien entendu, essentiell ement formative mais les retentissements sur les comportements devraient être davantage explorés...

\section{La formation des formateurs}

II s'agit là des animateurs de l'enseignement de base et de formation continue. O n conçoit qu'ils doivent avoir déà effectué l'enseignement de base ou un équivalent. Ils doivent, en plus, être rompus aux méthodes d'animation de groupe centrées sur l'analyse de $\operatorname{cas}^{77,78}$. Ils doivent, enfin, avoir une excellente connaissance du terrain, étant eux-mêmes cliniciens de préférence ${ }^{79}$.

\section{La formation de chercheurs en éthique médicale}

La recherche en éthique médicale est récente, en tant que tel. Elle emprunte ses méthodes à de nombreuses disciplines ${ }^{80}$. O n conçoit que le chercheur dans ce domaine ne soit pas obligatoirement focalisé sur l'éthique clinique et qu'il puisse provenir de l'une des nombreuses disciplines concernées. II doit posséder une bonne connaissance des méthodes utilisées et du terrain médical clinique et technique, objet de ses travaux. II doit pouvoir ainsi dialoguer de manière utile avec le corps de santé concerné à propos des grandes thématiques de l'éthique médicale (procréation, patrimoine génétique, interventions sur le corps humain, interventions sur la personnalité, fin de vie et mort, conséquences des actions médical es sur l'environnement etc.).

\section{Difficultés et limites}

Si l'enseignement del'éthiquemédicalea toujours une certaine difficulté à se situer, c'est en grande partie du fait de ses limites et difficultés. Trois d'entre elles nous paraissent majeures.

\section{En premier lieu, l'ancrage de l'éthique}

Celui-ci est délicat puisqu'elle concerne toutes les disciplines médical es et de nombreuses disciplines non médicales qui peuvent légitimement revendiquer son appartenance. Si l'éthique concerne tous les savoirs, l'éthique médicale, et, particulièrement l'éthique clinique pratique, concerne directement et en premier lieu le médecin puisqu'il est prescripteur. Pour éviter les conflits de valeur liés aux horizons idéologiques non homogènes, il nous semble naturel de voir confier la coordination d'un tel enseignement à des formateurs médecins. $M$ ais il doit bien s'agir d'une coordination, faisant place aux autres disciplines concernées, et ouvrant ainsi largement le débat médical. Au sein de la formation médicale, l'enseignement peut demeurer disséminé dans toutes les disciplines concernées, laissant la coordination à une personnalité dont la compétence en la matière s'est affirmée au fil du temps. C'est le schéma le plus habituel. II serait 
cependant plus logique de rattacher l'enseignement de l'éthique médicale à celui de la thérapeutique dont elle fait directement partie. M ais cette position n'est facilement acceptable que dans les pays, peu nombreux, qui possèdent une discipline thérapeutique individualisée. Du fait de l'importance de l'éthique en formation médicale, l'idéal serait de voir s'individualiser une disciplineà part entière qui aurait pour mission, non seulement la promotion de son enseignement, mais aussi le développement de la recherche en la matière ${ }^{91}$.

\section{En second lieu, la compétence des formateurs}

et leur nombre représentent une autre difficulté non négligeable. Pour transférer le savoir factuel et conceptuel, ces formateurs peuvent appartenir à toutes les disciplines concernées. En matière de savoir procédural et décisionnel, ces formateurs, pour des raisons de crédibilité, doivent, de préférence, appartenir au monde des praticiens cliniciens expérimentés. IIs doivent aussi posséder une bonne expérience des méthodes pédagogiques actives. On voit là se dessiner une spécialisation qui justifie largement I'autonomisation d'une discipline «éthique clinique et aide à la décision » en médecine ${ }^{82}$.

\section{En dernier lieu, les moyens alloués}

à un tel enseignement sont tout aussi déterminants. IIs sont indispensables à l'élaboration de base de données pratiques, à l'ouverture nécessaire aux disciplines non médicales, à la formation des formateurs médicaux, à la structuration d'évaluations valides. Il s sont, enfin, indispensables au soutien d'une recherche active qui, seule, peut permettre une évaluation adaptative pour l'avenir. Ici, ces moyens ne peuvent, pour l'essentiel, provenir que $d^{\prime}$ une prise de conscience institutionnelle et d'une volonté politique affichée.

\section{L'éthique de l'enseignement}

L'éthique de l'enseignement médical représente un autre thème de réflexion signalé ${ }^{35}$ mais ne soulève pas les mêmes difficultés que celles rencontrées à propos del'enseignement de l'éthique. En effet, les conflits de valeur peuvent facilement trouver une solution, ici, dans une

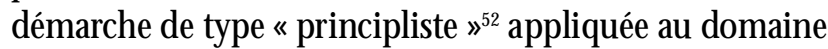
de l'enseignement. Elle est alors dominée par le respect de val eurs dont, essentiellement, l'indépendance et la justice ainsi que par la définition du champ des responsabilités. C'est plus la régulation de ces normes de qual ité qui pose problème, puisque, là encore, l'éthique de l'enseignement représente la démarche qui doit nous indiquer le meilleur chemin à prendre dans une optiqued'optimisation de l'action pédagogique.

\section{Les valeurs de base}

\section{La liberté de l'enseignement}

Elle représente, certainement, la valeur fondamentale dont les autres découlent. La liberté de l'enseignement ne consiste pas à enseigner n'importe quoi, mais à privilégier les certitudes scientifiques et les notions utiles dans le cadre d'objectifs institutionnels clairement définis et affichés. Ici, il s'agit de former des médecins et personnels de santécompétents, c'est-à-dire, susceptibles d'aider la prise des décisions les meilleures dans le soin des malades, respectant les faits scientifiques, les sensibilités des soignés et les nécessités collectives. D ans cette approche, l'enseignement doit être libre de toute contrainte personnelle, administrative ou politique L'application de cette valeur, érigée en principe d'indépendance, fait référence aux conflits d'intérêts possibles qui doivent être repérés et résolus et qui peuvent être nombreux (liens avec l'industrie pharmaceutique, recrutement personnel de patients, liens avec un certain pouvoir syndical ou politique en formation continue... ). L'application de ce principe nécessite honnêteté, c'est-à-dire, sincérité et objectivité dans le choix et la présentation de l'information pédagogique. En cas de conflit d'intérêt, la meilleuresolution serait de refuser la mission ou, au moins, d'afficher nettement leconflit pour en informer les personnes concernées.

\section{La justice de l'enseignement}

II sagit, ici, du principe de «justice scolaire » qui singularisel'égal ité des chances en matière de savoir. Ce principe, différent de la justice distributive ${ }^{33}$, est axé sur l'équité qui cherche à respecter toutes les chances de toute personne en formation, sans discrimination à partir d'autres critères que ceux déterminés, au préalable, pour permettre la meilleure progression possible de chacune d'elles. Différent de la rigueur, il impose également le respect des normes et règlements institutionnels dans le cadre de l'impartialité (application de normes valides identiques à tous les formés). L'application de ce principe, loin de conduire au laxisme, est plus à l'origine d'une définition de la discipline commeétant représentée par l'application stricte de règles consensuelles, établies et acceptées de tous au préalable à toute action pédagogique. 


\section{Références}

\section{La responsabilité des enseignants,}

\section{des formés et des structures d'enseignement}

Cette troisième val eur définit les limites des devoirs et obligations de chacun 24,84. La responsabilité des enseignants leur commande de mettre en œuvre leur conscience pour travailler dans le cadre de la qualité optimale due aux formés et, par extension, aux malades dont ceux-ci auront la charge. La responsabilité des enseignants ne peut s'entendre que dans le cadre des applications à obtenir une appropriation correcte des savoirs pour permettre l'autonomie d'action des formés. Au-delà de cette appropriation, la responsabilité est celle des formés qui doivent également tout mettre en œuvre pour acquérir correctement et appliquer avec compétence les savoirs appropriés. La responsabilité de la collectivité, à travers l'institution, s'exprime par une obligation de moyens: obligation de mettre en œuvre les moyens appropriés à la réal isation satisfaisante des objectifs institutionnels affichés. On le voit, le principe de responsabilité appliqué à l'enseignement doit conduire à l'établissement d'un contrat tripartite institution-formateur-formé, contrat qui doit définir les devoirs et obligations respectifs.

\section{Les systèmes d'application et de régulation}

L'application et la régulation de ces normes est délicatecar elle a tendance souvent à dévier vers une tendance à l'inquisition ou à la manipulation démagogique. Elle est assurée par trois démarches possibles.

\section{La première fait référence à la notoriété}

Ell représente une régulation naturelle et historique. Souvent mais non nécessairement liéeà la qualité, la notoriété peut être à l'origine de débordements où s'expriment largement les conflits d'intérêt. Elle ne peut donc apparaitre comme suffisante.

\section{L'évaluation formative}

Elle représente la démarche la plus rationnelle. Bien utilisée, elle est susceptible d'apporter d'excellents arguments pour conduire un changement adapté, s'il apparaît nécessaire. Elle est cependant soumise au bon vouloir des responsables d'enseignement et devrait demeurer confidentielle (communiquée aux seules personnes concernées) pour éviter l'apparition d'une concurrence malsaine. Elle gagnerait toutefois à se développer et ses méthodes doivent figurer au programme de toute formation de formateurs cohérente.

\section{L'évaluation sommative et les instances de contrôle}

A côté de l'évaluation formative existe, de manière plus ou moins officialisée, une évaluation sommative. Ellene peut, en fait, opérer valablement que sur des critères de jugements normatifs prédéfinis qui incitent parfois à la délation et la fait regarder comme une instance disciplinaire. En effet, les instances de contrôle, essentiellement administratives, ont tendance à privilégier l'opinion des formés et à entraîner, de fait, des dérives vers une certaine forme de vengeance ou de démagogie en cas de sanction. Ce type d'évaluation est plus recevable lorsqu'elle est réalisée par des pairs et préalablement acceptée par tous comme la règle du jeu...

\section{Conclusions}

S'inscrivant dans une démarche de qualité, l'enseignement de l'éthique doit tenir compte des exigences des publics concernés. Un minimum indispensable peut, maintenant, être défini et adaptéaux besoins locaux. Sa mise en œuvre est encore gênée par des questions de coordination et d'évaluation qui peuvent trouver des solutions si les intentions et objectifs de cet enseignement sont clairement définis et affichés. À ce minimum doit être associé une démarche modulaire susceptible de répondre aux besoins spécifiques de la formation continue, de celle des formateurs et des chercheurs. L'éthique de l'enseignement, centrée sur trois valeurs fondamentales, doit trouver son épanouissement dans sa libre acceptation par les acteurs concernés que sont les enseignants, les formés et l'institution. À ce prix, l'éthique apparaît comme un élément indispensable et incontournable de la formation médicale qui devrait, par son intermédiaire, non seulement s'enrichir d'une approche pluridisciplinaire, mais aussi évoluer dans le cadre de la recherche de la qual ité optimale. 


\section{Références}

1. G uyatt GH, Sackett D L, Sindair JC, H ayward R, Cook DJ, Cook RJ. for the Evidence Based M edicineW orking Group. U sers guides to the medical lierature IX. A method for grading health care recommandations. J A M. A $1996 ; 274: 1800-04$.

2. Wyatt J. U se and source of medical Knowledge. Lancet $1991 ; 338: 1368-73$.

3. H ottois G. Bi oéthique. In H ottois G, M issa JN (eds) : Nouvelle encyclopédie de bioćthique. Bruxelles: D eBoeck Universitééditions, 2001 : 124-31.

4. Leber G. Ethique clinique. In H ottois G., M issa J.N . (eds) : N ouvelle en cyclopédie de bioéthique. Bruxelles: D eBoeck Université éditions, 2001 : 393-97.

5. Hervé C. Enseignement. In Hottois G, M issa JN (eds) : Nouvelle encyclopédiedebioéthique. Bruxelles: D eBoeck Universitééditions, 2001 : 383-86.

6. Loewy EH , Loewy RS. Bioethics at the crossroad. H ealth CareAnal. $2001 ; 9(4):$ 463-77.

7. Llorca G. Rôle des Comités d'éthique dans le formation médicale. Pédagogie M édicale. $2001 ; 2$ : 157-62.

8. Angel os P, D aRosa D A, D erossis AM, Kim B. M edical ethics curriculum for surgical residents: results of a pilot project. Surgery $1999 ; 126(4)$ : 701-5.

9. Beauchamp G. General surgeons and dinical ethics: a survey. Can J Surg. $1998 ; 41(6): 451-4$.

10. Coughlin SS, Katz WH, M attison DR. Ethics instruction at schools of public health in the U nited States. Association of Schools of Public Health Education Committee Am J Public H ealth 1999 ; 89(5) : 76870.

11. D ickenson D L, Parker M J. The European Biomedical Ethics Practitioner Education Project : an experiential approach to philosophy and ethics in health care education. M ed H ealth Care Philos $1999 ; 2$ (3) : 231-7.

12. D owning M T, Way DP, Caniano DA. Results of a national survey on ethics education in general surgery residency programs. Am J Surg 1997 ; 174(3) : 364-8.

13. Goldie J, Schwartz L, M cC onnachie A, M orri son J. Impact of a new course on students potential behaviour on encountering ethical dilemmas. M ed Educ 2001 ; 35(3) : 295-302.

14. M iyasaka M, Akabayashi A, Kai I, O hi G. An international survey of medical ethics curricula in Asia. J M ed Ethics 1999 ; 25(6) : 514-21.
15. M usick DW. Teaching medical ethics: a review of the literature from $\mathrm{N}$ orth American medical schools with emphasis on education. M ed Health CarePhilos 1999 ; 2(3) : 239-54.

16. Parizeau M H . Les F ondements de la bioéthique. Bruxelles, M ontréal : Édition D eB oeck/ERPI, 1992.

17. Rameix S. Fondements philosophiques de l'éthique médicale. Paris: Édition Ellipses, 1996.

18. Jonsen $A$. and Toulmin S. Theabuse of Casuistry : a his tory of M oral Reasoning. Berkeley : University of California Press, 1988.

19. Parizeau M H . : Bi oéthique. Ethique appliquée. In : Canto-Sperber M . Dictionnaire d'éthique et de philosophie morale. Paris: Éditions Presses U niversi taires de France, $2^{\circ}$ édition 1997 : 155-160 \& 534-40.

20. Reich W (ed.). Encyclopedia of Bioethics N ew York: 2nd edition, 5 vols, Simon \& Schuster/M cmillan, 1995.

21. Russ $S$ W. Tackling ethical dilemmas in personality assessment. J PersAssess $2001 ; 77(2):$ 255-8.

22. Gross M L. M edical ethics education : to what ends? J Eval Clin Pract $2001 ; 7(4)$ : 387-97.

23. Ashcroft R E. Teaching for patient-centred ethics $M$ ed $H$ ealth Care Philos $2000 ; 3(3):$ 287-95.

24. Llorca G. La formation médicale. Aspects conceptuels Lyon : M éditions, 1999.

25. Tardif J. Pour un enseignement stratégique. L'apport de la psychologie cognitive. M ontréal : Les Éditions Logiques, 1997.

26. D auber $F$ W. Critical Thinking: An Introduction to Reasoning. N ew York : Barnesand N oble,1996 : 37-45.

27. M arckmann $G$. Recommendations for the ethical deve lopment and use of medical decision-support systems. M ed Gen M ed $2001 ; 3(3): 5$.

28. Self $D J$, Olivarez $M, B$ aldwin $D C$. The amount of small-group case-study discussion needed to improve moral reasoning skills of medical students. Acad M ed $1998 ; 73(5): 521-3$.

29. $H$ abermas J. M orale et communication. Traduction Française, Paris: LesÉditions du Cerf, 1986.

30. Patenaude J, Lambert C, Dionne M, M arcoux H . L'éthique comme compétence clinique: modélisation d'une approche dialogique. Pédagogie M édicale 2001 ; $2: 71-9$. 


\section{Références}

31. Jonsen A R, Veatch R, Walters L. Source B ook in Bioethics: A D ocumentary H istory. Washington, D.C : Georgetown U niversity PressEds 1998.

32. Lenoir N, M athieu B. Les normes internationales de la bi oéthique. Paris: Éditions Presses U niversitaires de France, 1998.

33. Ger B, Culver C M, Clouser K D. Bioethics: A Return to Fundamentals $\mathrm{N}$ ew York : O xford Universty Press, 1997.

34. D eG razia D. «M oving Forward in Bioethical Theory: Theories, Cases, and Specified Principlism». J M edic Philosophy $1992 ; 17: 511-39$.

35. M arcoux $\mathrm{H}$, Patenaude J. L'éthique et la formation médicale. 0 ù en sommes-nous? 0 ù allons-nous. Pédagogie M édicale $2000 ; 1: 23-30$.

36. Strong C. "Justification in Ethics". In M oral Theory And $M$ oral Judgments in M edical Ethics, B. Brody. D ordrecht (ed), The N etherlands: Kluwer Academic Publishers, 1988 : 193-211.

37. Yarborough $M$, JonesT, Cyr T A, PhillipsS, Stetzner D. Interprofessional education in ethicsat an academic health sciencescenter. Acad M ed 2000 ; 75(8) : 793-800.

38. Diekema D S, Shugerman R P. An ethicscurriculum for the pediatric res dency program. Confronting barriers to implementation. Arch Pediatr Adolesc M ed 1997 ; 151(6) : 609-14.

39. Braunack-M ayer A J, Gillam L H, Vance E F, Gillett G $R$, Kerridge I $H, M C P$ hee J, Saul $P$, Smith $D E$, Wellsmore H M , Koczwara B, RogersW A, M cNeill P M, N ewell C J, Parker M H, Walton M, Whitehall J S. An ethics core curriculum for Australasian medical Schools M ed J Aust. 2001 ; 175(4) : 205-10.

40. M arcoux H, Lamontagne C, C ayer S, D esrochers A, Gauthier D. La formation en éthique. Identification des besoins par des médecins enseignants en médecine familiale Can Fam Physician 2001 ; 47 : 1208-15.

41. Llano A. M edical ethics education in Colombia. Med Law $2000 ; 19(3): 415-23$.

42. Gross M L. Ethicseducation and physician morality. Soc Sci M ed 1999 ; 49(3) : 329-42.

43. Carter $B$ S, Roberts A, M artin R, Fincher R M . A longitudinal ethics curriculum for medical students and generalist residents at the $M$ edical $C$ ollege of $G$ eorgia. Acad M ed 1999 ; 74(1 Suppl) : S102-3.

44. Robin M L, Caniano D A. Analys sof dinical bioethics teaching in pediatric surgery residency. J Pediatr Surg $1998 ; 33(2)$ : 373-7.
45. Fulford K W, Yates A, H ope T. Ethics and the G M C core curriculum : a survey of resources in UK medical schools. J M ed Ethics 1997 ; 23(2) : 82-7.

46. Silverman $H$ J. D escription of an ethics curriculum for a medicine residency program. West J M ed 1999 ; 170(4) : 228-31.

47. Layman $\mathrm{E}$. Ethics education : curricular considerations for the allied health disciplines J Allied H ealth 1996 ; 25(2) : 149-60.

48. Lazarus C J , Chauvin SW , Rodenhauser P , Whitlock R. T he program for professional values and ethics in medical education. Teach Learn M ed $2000 ; 12(4)$ : 208-11.

49. Feuillet-Le-M intier $B$. Ethics education in medical schools: the role of jurists. M ed Law $2000 ; 19(3)$ : 403-7.

50. ColesR. Themoral education of medical students. Acad M ed $1998 ; 73(1): 55-7$.

51. Engelhardt $\mathrm{T}$. : The Fondations of Bioethics. $\mathrm{N}$ ewYork : O xford University Press Edition, 1986.

52. Beauchamp T., Childress J. Principles of Biomedical Ethics N ew York : O xford U niversity PressE dition, 4th edition, 1994.

53. Veatch R M. M edical ethics 2nd edition, N ew York: Bartlett and Jones edition, 1994.

54. Jonsen A R, Siegler $M$, Winsade W J. Clinical ethics New York : M CG raw-H ill edition, 4th edition, 1998.

55. Canto-Sperber M . D ictionnaire d'éthique et de philosophie morale. Paris: Éditions Presses U niversi taires de France. $2^{\circ}$ édition 1997.

56. H ottois G, M issa J N . N ouvelle encyclopédie de bioé thique Bruxelles: D eB oeck U niversité Éditions, 2001.

57. Walters $L, K a h n T J$ (eds.). Bibliography of Bi oethics. Washington, DC : Georgetown U niversity. Published annualy. O n-line as Bioethicsine, National Library of $M$ edicine $M$ edlars

58. Réseau ROD IN . Information et diffusion desconnais sancesen éthique médicale "http//www.inserm.fr/ethique, 1999.

59. Carson A M. That's another story: narrative methods and ethical practice. J M ed Ethics $2001 ; 27(3)$ : 198202.

60. N eitzkeG. Teaching medical ethicsto medical sudents: moral, legal, psychological and philosophical aspects. M ed Law 1999 ; 18(1) : 99-105.

61. N icholas B, Gillett G. D octors stories, patients stories: a narrative approach to teaching medical ethics J $M$ ed Ethics 1997 ; 23(5) : 295-9. 
62. Tysinger J W, Klonis L K, Sadler J Z, Wagner J $M$. Teaching Ethi cs using Small-G roup, Problem-based Learning. J M ed Ethics, 1997 ; 23(5) : 315-318.

63. D es $M$ archais]. Apprendre à devenir médecin. Bilan d'un changement pédagogique centré sur l'étudiant. Canada : U niversité de Sherbrooke édition, 1996.

64. Lipman A J , SadeR M , Glotzbach AL. Application of a web-based instruction for a clinical course Acad M ed $1999 ; 74(5): 571-2$.

65. Lurie N . Preparing physicians for practicein managed careenvironments Acad M ed $1996 ; 71$ (10) : 1044-49.

66. Collins J, Amsal S, Alderson P O. 2001 Joseph E. and $\mathrm{N}$ ancy $\mathrm{O}$. Whitley Award. Teaching noninterpretive skills to radiology residents: a collaborative effort between the American College of Radiology and the Association of Program Directors in Radiology. Acad Radiol 2001 ; 8(9) : 903-7.

67. Savulescu J, Crisp R, Fulford KW, H opeT. Evaluating ethics competence in medical education. J M ed Ethics 1999 ; 25(5) : 367-74.

68. Brajenovic-M ilic B, Ristic S, Kern J, Vuletic S, Ostojic $S$, Kapovic $M$. The effect of a compulsory curriculum on ethical attitudes of medical students. Coll Antropol $2000 ; 24(1): 47-52$.

69. Hayes R P, Stoudemire A, Kinlaw K, Dell M L, Loomis A. Q ualitative outcome assessment of a medical ethics program for dinical clerkships: a pilot study. Gen H osp Psychiatry. $1999 ; 21(4)$ : 284-95.

70. Sulmasy DP, M arx E S. Ethics education for medical house officers: long-term improvements in knowledge and confidence. J M ed Ethics $1997 ; 23(2)$ : 88-92.

71. Roberts], N orman G. Reliability and learning from the $O$ bjective Structured Clinical Examination. M edical Education 1990, 24 : 219-23.

72. Singer PA, Robb A, Cohen R, N orman G , Turnbull J. Performance-based assessment of dinical ethics using an objectivestructured clinical examination. Acad M ed 1996 ; 71(5) : 495-98.
73. Charlin B, Gagnon R, Sibert L, Van der V leuten C. Le test de concordance de script, un instrument d'évaluation du raisonnement clinique. Pédagogie M édicale, soumis pour publication.

74. N ilstun T, Cuttini M, Saracci R. Teaching medical ethics to experienced staff : partici pants, teachers and method. J M ed Ethics2001 ; 27(6) : 409-12.

75. Braxton JM , Baird LL. Preparation for professional seffregulation. Sci EngEthics $2001 ; 7(4): 593-610$.

76. Kenny N, Sargeant J, Allen M. Lifelong learning in ethical practice : a challenge for continuing medical education. J Contin Educ Health Prof $2001 ; 21(1)$ : 24-32.

77. Schuklenk U. Ethical issuesin continuing professional development. S Afr M ed J $2001 ; 91(11)$ : 955-57.

78. M ucchielli R. Lesméthodesactives dansla pédagogiedes adultes. Paris: LesÉditionsESF, 1998.

79. Fraser HC, Kutner JS, Pfeifer M P. Senior medical students perceptions of the adequacy of education on endof-life issues Palliat M ed 2001 ; 4(3) : 337-43.

80. Sugarman J, Sulmasy D P. M ethods in medical ethics. Washington D.C : Georgetown U niversity press edition, 2001.

81. Jonsen AR. "Bioethics as a discipline". In The Birth of Bioethics. Oxford : Oxfrod U niversity Press edition, 1998 : 325-51.

82. Parizeau M H. Ethicien. In H ottois G, M issa JN . $N$ ouvelle en cyclopédie de bi oéthi que. Bruxelles: D eBoeck U niversitééditions, 2001 : 391-93.

83. Rawls J. A Theory of Justice. Cambridge, M A : $\mathrm{H}$ arvard U niversity Press edition, 1971 et traduction française: Théorie de la justice. Paris: Le Seuil Éditions, 1987.

84. Jonas H . Le principe responsabilité. Paris: Cerf Éditions, $3^{\circ}$ édition, 1993. 